\title{
Geometric Homogeneity and Controllability of Nonlinear Systems
}

\author{
Patricio A. Vela ${ }^{\dagger \ddagger}$, Joel W. Burdick ${ }^{\ddagger}$ \\ ${ }^{\dagger}$ Control and Dynamical Systems \\ ${ }^{\ddagger}$ Mechanical Engineering \\ California Institute of Technology, Mail Code 107-81, Pasadena, CA, 91125 \\ pvela@cds.caltech.edu, jwb@robotics.caltech.edu
}

\begin{abstract}
We followup on a suggestion from Bullo and Lewis [1] concerning the importance of geometric homogeneity for mechanical systems. It is shown that controllability results for a large class of mechanical systems with drift can be recovered by considering a class of nonlinear dynamical systems satisfying certain homogeneity conditions.
\end{abstract}

\section{Introduction}

Mechanical control systems form a large and important subclass of nonlinear control systems. Besides their practical utility, mechanical systems also have inherent structure that simplifies their analysis. Determining controllability for nonlinear control systems is in general quite difficult. Sussmann [2] provides sufficient conditions to determine small-time local controllability, however there is factorial growth in the number of elements to test. Lewis and Murray [3] showed how the affine connection description of mechanical systems prunes the tests found in [2] for the particular case of configuration controllability. Their work is applied to simple mechanical systems, which are characterized by Lagrangians with kinetic and potential energy terms only; constraints may be included. In [1], Bullo and Lewis demonstrate that geometric homogeneity is behind much of the simplifications. They further argued that geometric homogeneity should be a fruitful avenue of further study for nonlinear control systems. This is especially true given that analogous controllability results have subsequently been found to hold for alternative approaches to mechanical control systems $[4,5,6]$. This paper develops the concept of geometric homogeneity for vector bundles and demonstrates how geometric homogeneity is the connecting link to all of these seemingly related results.

Section 2 reviews and develops homogeneity for application to homogeneous control systems. These methods are used to extend the configuration controllability theorems of Lewis and Murray to a generic vector bundle in Section 3. These results are applied in Section 4 to various control sytems evolving on vector bundles. As all of the instances have been studied in the literature, the analysis demonstrates how they all may be placed within a common framework.

\section{Geometric Homogeneity and Vector Bundles}

Geometric homogeneity has been used in various studies of nonlinear dynamics and control. Controllability results often use dilations [2,7]. Mc'Closkey and Morin [8] use homogeneity to obtain stabilizing controllers for certain nonlineas systems with drift. Crampin [9] uses homogeneity to study the geometry of Lagrangian systems. We seek to develop the

\footnotetext{
${ }^{1}$ This work was supported in part by the National Science Foundation by Engineering Research Center grant NSF9402726.
}

relevant ideas concerning geometric homogeneity in a more systematic manner. We first review relevant concepts and terminology from differential geometry [10].

Vector Bundles. A (differentiable) fibre bundle $(Q, \pi, M, F)$ consists of: (1) a differentiable manifold $Q$ called the total space (configuration space), (2) differentiable manifold $M$ called the base space, (3) differentiable manifold $F$ called the fibre, and (4) a surjection $\pi: Q \rightarrow M$ called the projection, together with compatibility conditions that will not be discussed. The fibre $\mathrm{F}$ is diffeomorphic to $\pi^{-1}(q)$, where $q \in M$. Given a fibre bundle $\pi: Q \rightarrow M$, a section $\sigma: M \rightarrow Q$ is a smooth map satisfying $\pi \circ \sigma=\mathrm{id}_{M}$. The space defined by the collection of all sections on the fiber bundle $Q$ is denoted by $\Gamma(Q)$. It is itself a fiber bundle.

A vector bundle $(E, \pi, M, V)$ is fiber bundle whose typical fiber is a vector space. Given a vector bundle $\pi: E \rightarrow M$, the zero section, denoted by $\sigma_{0}$, is a smooth mapping which maps points in the base space to the zero vector,

$$
\sigma_{0}(x)=0_{x} \in E_{x}, \quad \forall x \in M .
$$

As a vector space, the image of the zero section $M \times\{0\}$ is isomorphic to the the base space, $M$. The manifold structure of $E$ is obtained from local charts $(U, \psi)$, which are also called local trivializations. In a local trivialization, the vector bundle is a direct product space, $\psi(U) \subset M \times V$. Often, when giving coordinate representations, we will simply refer to a local trivialization as $E \cong M \times V$.

If two bundles share the same base space, $M=M_{1}=M_{2}$, they may be composed via the fiber product, denoted $\times_{M}$, to form a new bundle $Q_{1} \times{ }_{M} Q_{2}$. Let vector bundles $E_{1}$ and $E_{2}$ have a common base space. Their fiber product, Whitney sum, is denoted $E_{1} \oplus E_{2}$. In a local trivialization, the Whitney sum is, $E_{1} \oplus E_{2} \cong M \times V_{1} \times V_{2}$.

Let the tangent space, $T M$, to $M$, have the local trivialization, $T M \cong M \times W$, where $W$ models the tangent fiber of $M$. The fiber of the tangent space to a vector space can be modelled by the vector space itself, $T V \cong V \times V$. The local trivialization to the tangent space $T E$, can be given by, $T E \cong M \times V \times W \times V$, where $M \times V$ represents the vector bundle, and $W \times V$ represents the tangent fiber to the vector bundle. The trivialization suggests two projection operators for $T E, \rho_{1}$ and $\rho_{2}$. The operator $\rho_{2}$ is only locally defined (it depends on the local trivialization). In a local trivialization, $\rho_{1}$ and $\rho_{2}$ operate as follows,

$$
\begin{aligned}
& \rho_{1}(q, u, w, v)=(q, u, w, 0), \text { and } \\
& \rho_{2}(q, u, w, v)=(q, u, 0, v) .
\end{aligned}
$$

Locally, $\rho_{1}=\mathrm{id}_{T E}-\rho_{2}$. 
Geometric Homogeneity. Much of the terminology concerning geometric homogeneity can be found in Crampin [9] or Kawski [7]. Geometric homogeneity is defined using the dilation operator $\delta_{t}$, which dilates the vector fiber,

$$
\delta_{t}: E \rightarrow E ; \quad(x, u) \mapsto\left(x, e^{t} u\right) .
$$

The dilation operator satisfies $\left(\delta_{t}\right)^{p}=\delta_{p t}$. Corresponding to the dilation is its infinitesimal generator, $\Delta$, a vector field on $E$. In a local trivialization, the generator is,

$$
\Delta=u^{i} \frac{\partial}{\partial u^{i}}
$$

An alternative to the dilation operator exchanges the additive property of the dilation with a multiplicative one,

$$
\tilde{\delta}_{c} \equiv \delta_{\ln (c)}: E \rightarrow E ; \quad(x, u) \mapsto(x, c u)
$$

Our working definition of homogeneity follows.

Definition 1 A mapping between bundles $\Psi: E_{1} \rightarrow E_{2}$ is homogeneous of order $p$ if $\Psi \circ \delta_{t}=\delta_{p t} \circ \Psi$.

The two bundles need not have the same base nor fiber. We assume that $\Psi$ is a smooth function defined over $E$, and that all vector bundles are finite dimensional. Below, $E_{1}$ and $E_{2}$ are vector bundles.

Proposition 1 [11] All smooth functions, $\Psi: E_{1} \rightarrow E_{2}$ of homogeneous order 0 are equivalent to mappings of the zerosection on $E_{1}$ into $E_{2}$.

Proposition 2 [10] The only smooth functions, $\Psi: E_{1} \rightarrow$ $E_{2}$ of homogeneous order $-p$ for $p>0$ are mappings of the zero section on $E_{1}$ to the zero-section on $E_{2}$.

Proposition 3 [1]] lf $\Psi: E_{1} \rightarrow E_{2}$ is homogeneous of order $p \geq 1$, then $\Psi$ evaluated on the zero section of $E_{1}$ results in the zero section of $E_{2}$.

Note that configuration controllability evaluations are made around the zero section of a vector bundle; the above homogeneity results will allow certain vector fields to be ignored. This is precisely how Lewis and Murray [3] achieved a simplification of Sussmann's controllability theorem.

The Tangent Bundle and Vector Fjelds. We focus now on the geometric homogeneity properties of the tangent bundle $T E$, and of the space of vector fields $\mathcal{X}(E)$. Using the projection operator there is a natural notion of vertical for $T E$.

Definition 2 The vertical bundle over $E$, denoted by $V E$, is the subbundle of $T E$ given by the union of $T \pi^{-1}\left(0_{q}\right)$ for all $q \in Q$. A vector in $T E$ is vertical if it lies in the kemel of $T \pi$.

Definition 3 There is a canonical isomorphism between $E \times_{M} E$ and $V E$, called the vertical lift. It is given by,

$$
v^{\mathrm{lift}}=\left.\frac{\mathrm{d}}{\mathrm{d} t}\right|_{t=0} \cdot\left(u_{x}+t v_{x}\right), \quad u, v \in E .
$$

A complementary horizontal subbundle can be defined using a connection form, also called an Ehresmann connection.

Definition 4 An Ehresmann connection, $A$, is a vertical val. ued one form on a manifold $Q$ that satisfies,

1. $A_{q}: T_{q} Q \rightarrow V_{q} Q$ is a linear map for each point $q \in Q$.

2. $A$ is a projection, $A \circ A=A$.
The horizontal subbundle HE is equal to the kernel of the Ehresmann connection. The natural projection operator, $\rho_{2}$, locally defines a trivial connection on $T E$.

$$
A^{\text {triv }}: T E \rightarrow V E, \quad A^{\text {triv }}=\rho_{2} \text {. }
$$

This locally defined trivial connection will be evaluated on the zero section according to the local controllability tests to be defined. Horizontal vectors are isomorphic to vectors on the tangent bundle of the base space via the bundle projection, $T \pi \cdot$

Definition 5 The vertical subspace $\mathcal{X}^{V}(E) \subset \mathcal{X}(E)$, consists of all vector fields that are vertical for all points in $E$.

Proposition 4 If $X, Y \in \mathcal{X}^{V}(E)$, then $[X, Y] \in \mathcal{X}^{V}(E)$.

The notion of homogeneity extends to vector fields via $\Delta$.

Definition 6 A vector field $X \in \mathcal{X}(E)$ is said to be homogeneous of order $p$ if, $[\Delta, X]=p X$, for $p>-2$.

Under the trivial splitting of vector fields into horizontal and vertical, the dilation vector field has the following properties,

Proposition 5 [11] Let $\Delta$ be the infinitesimal generator cor responding to the dilation action, $\delta_{t}$. Given a vector field $X \in \mathcal{X}(E)$, the following pmperties hold:

1. $\left[\Delta, X^{H}\right]$ is horizontal, and

2. $\left[\Delta, X^{V}\right]$ is vertical,

for the trivial decomposition, $X=X^{H}+X^{V}$, of $X$ into horizontal and vertical components, respectively.

Proposition 6 Given $X, Y \in \mathcal{X}(E)$ homogeneous of order $p$ and $q$, respectively, $[X, Y]$ is homogeneous of order $p+q$.

Proposition 7 [11] Any mapping $\Psi: E \rightarrow$ E preserving the base, i.e., $\pi=\pi \circ \Psi$, and homogeneous of order $p$, loses one degree of homogeneity when lifted via $\mathrm{Eq} .(5)$, i.e., $\Psi^{\text {lift }}$ is homogeneous of order $p-1$.

Corollary 1 Given a section of the vector bundle $E$, its vertical lift is homogeneous of order -1 .

As stated next, the converse to the corollary also holds.

Proposition 8 [9] All vector fields of homogeneous order -1 are the vertical lift of a section of $E$.

Proposition 9 [11] Using the trivial horizontal connection, the decomposition of the vector field $X=X^{H}+X^{V}$ of homogeneous order $p$ has the following properties:

1. $X^{H}$ is in 1-1 correspondence with $Y \in C^{\infty}(E, T M)$ where $Y$ is homogeneous of order $p$.

2. $X^{V}$ is in 1-1 correspondance with $\Psi^{\mathrm{lift}}$, where $\Psi \in$ $C^{\infty}(E, E)$ is homogeneous of order $p+1$.

This is analogous to the discussion following Eq. (3.1) in [1].

Corollary 2 For $X, Y \in \mathcal{X}(E)$ vertical lifts, $[X, Y]=0$.

The Jacobi identity implies a symmetry of the following bracket construction.

Corollary 3 If $X, Y \in \mathcal{X}(E)$ are vertical lifts, then $[X,[\Gamma, Y]]=[Y,[\Gamma, X]]$, for any $\Gamma \in \mathcal{X}(E)$.

Via Corollary 3 , the $(2,1)$-tensor $[\cdot,[\Gamma, \cdot]]$ may be used to define a symmetric product for lifted vector fields. 
Definition 7 The symmetric product of lifted vector fields using the vector field $\Gamma \in \mathcal{X}(E)$ is defined to be,

$$
\langle X: Y\rangle^{\Gamma} \equiv[X,[\Gamma, Y]],
$$

where $X, Y \in \mathcal{X}(E)$ lifted vector fields.

We will simply write $\langle X: Y\rangle$ without reference to the vector field $\Gamma$ when the context is clear. The symmetric product was originally derived and defined using the Riemmanian or affine connection structure of simple mechanical systems $[12,3]$, and is known to be derivable from the description above. The symmetric product, therefore, can be used in a more general setting. What is critical to simple mechanical systems is not the symmetric product alone, but the homogeneous structure inplied by the Lagrangian framework from which such systems are derived. The homogeneous structure will imply that it is only Jacobi-Lie brackets with a structure similar to the one above that will be important from both control theoretic and dynamical systems perspectives. This is made more explicit through the use of a gradation.

Gradations of Homogeneous Spaces. Define the vector subbundle of homogeneous order $k$ to be

$\mathcal{P}_{k} \equiv\{X \in \mathcal{X}(E) \mid X$ is of homogeneous degree $k$.$\} .$

Propositions 2 and 6 imply that: (1) $\left[\mathcal{P}_{i}, \mathcal{P}_{j}\right] \subset \mathcal{P}_{i+j}$; and (2) $\mathcal{P}_{k}=\{0\}, \forall k<-1$. Accordingly, we may define the following union of homogeneous spaces,

$$
\mathcal{M}_{k}=\oplus_{i=-1}^{i \leq k} \mathcal{P}_{i}
$$

which inherit the properties of its constitutive sets, (1) $\left\{\mathcal{M}_{i}, \mathcal{M}_{j}\right\} \subset \mathcal{M}_{i+j}$, and (2) $\mathcal{M}_{i}=\{0\}, \forall i<-1$. It can be seen that $\mathcal{M}_{-1}=\mathcal{P}_{-1}$, meaning that it is the most "basic" nontrivial space. Consequently, the spaces $\mathcal{M}_{k}$ form a gradation. For the systems that we will study, the vector field $\Gamma$ is restricted to not exceed homogeneous order 1, e.g. $\Gamma \in \mathcal{M}_{1}$. Therefore, $\left\langle X^{\text {lift }}: Y^{\text {lift }}\right\rangle^{\Gamma} \in \mathcal{M}_{-1}$ is again a lifted vector field. Most importantly, this implies that the symmetric product commutes with other lifted vector fields.

Definition 8 A nonlinear control system with drift is called a 1-homogeneous control system if the drift vector field is an element of $\mathcal{M}_{1}$ and the control vector fields are elements of $\mathcal{M}_{-1}$.

\section{Control of Dynamical Systems}

This core section of the paper develops the main controllability results for 1-homogeneous systems. We first review basic definitions for controllability of nonlinear affine control systems, as well as free Lie and symmetric algebras. Subsequently, the configuration controllability theorems analogous to those of Lewis and Murray [3] are presented.

We focus on nonlinear control systems in affine control form,

$$
\dot{z}=f(z)+g_{a}(z) u^{a}(t),
$$

where $f \in \mathcal{X}(E)$ is the drift vector field and the $g_{a} \in \mathcal{X}(E)$ are control vector fields. The variable $z$ evolves on the vector bundle $E$, and there are $m$ system inputs, $a=1 \ldots m$. The control inputs $u^{a}$ are piecewise constant.

Definition 9 The system (8) is controllable iffor any $z_{0}, z_{f} \in$ $E$, there exists admissable controls $u^{a}(t)$ such that the system evolving via (8) and starting at $z_{0}$ reaches $z_{f}$ in finite time.
Let $\mathcal{R}^{U}\left(z_{0}, T\right)$ denote the set of reachable points in $E$ at time $T>0$, starting at point $z_{0}$ and using admissable controls, $u^{a}(t)$, such that the trajectories remain in the neighborhood $U$ of $z_{0}$ for all $t \leq T$. Define,

$$
\mathcal{R}_{T}^{U}\left(z_{0}\right)=\bigcup_{0 \leq t \leq T} \mathcal{R}^{U}\left(z_{0}, t\right)
$$

Definition 10 The nonlinear control system (8) is locally accessible if for all $z \in E, \mathcal{R}_{T}^{U}(z)$ contains a non-empty open subset of $E$ for all $T>0$ and $U \subset E$.

Definition 11 A system (8) is small-time locally controllable (STLC) at $z_{0}$ if it is locally accessible at $z_{0}$ and if there exists $a T>0$ such that $z_{0}$ is in the interior of $\mathcal{R}_{t}^{U}\left(z_{0}\right)$ for every neighborhood $U$ of $z_{0}$ and $0 \leq t \leq T$. If this holds for any $z \in E$, the system is small-time Jocally controllable.

Configuration Controllability. If one is only interested in control of the base space $M$, and not of the vector fiber, then only configuration controllability of the system is needed, where the configurations are elements of $M$. Let $\mathcal{R C}^{U}\left(x_{0}, T\right)$ denote the set of reachable configurations in $M$ at time $T>$ 0 , starting at point $0_{x_{0}} \in E$ and using admissable controls, $u^{a}(t)$, such that the trajectories remain in the neighborhood $U$ of $x_{0}$ for all $t \leq T$. Define,

$$
\mathcal{R C}_{T}^{U}\left(x_{0}\right)=\bigcup_{0 \leq t \leq T} \mathcal{R C}^{U}\left(x_{0}, t\right)
$$

Definition 12 [3] A system (8) is locally configuration accessible at $x_{0} \in M$ if there exists a $T>0$ such that $\mathcal{R C}_{t}^{U}\left(x_{0}\right)$ contains a non-empty open set of $M$ for all neighborhoods $U$ of $x_{0}$ and all $0 \leq t \leq T$. If this holds for any $x_{0} \in M$, then the system is locally configuration accessible.

Definition 13 [3] A system (8) is small-time locally configuration controllable (STLCC) at $x_{0}$ if it is locally configuration accessible at $x_{0}$ and if there exists $a T>0$ such that $x_{0}$ is in the interior of $\mathcal{R C}_{t}^{U}\left(x_{0}\right)$ for every neighborhood $U$ of $x_{0}$ and $0 \leq t \leq T$. If this holds for any $x \in M$, then the system is called small-time locally configuration controllable.

Definition 14 The equilibrium subspace, $\mathfrak{E} \subset E$, of system (8) consists of the elements of the zero section of $E$ where $\dot{z}=0$.

Definition 15 [3] A system (8) is equilibrium controllable if, for $z_{1}, z_{2} \in \mathcal{E}$, there exists a solution $(c, u)$ of $(8)$ where $c$ : $[0, T] \rightarrow Q$ is such that $c(0)=\pi\left(z_{1}\right), c(T)=\pi\left(z_{2}\right)$, and both $c^{\prime}(0)=c^{\prime}(T)=0$.

The remaining subsections develop sufficient conditions for STLCC in our extended homogeneous context.

\subsection{Configuration Controllability revisited}

Here, we extend the work of [3] from simple mechanical control systems to the broader context of 1-homogeneous control systems. The resulting conditions apply to an enlarged class of systems, and reproduce many known results on controllability of mechanical or nonlinear affine control systems. The content and structure found in Sussmann [2], and Lewis and Murray [3], is assumed familiar to the reader. We develop our ideas using the same language and construction.

The standard control form will be rewritten as,

$$
\dot{z}=X_{S}+Y_{a}^{\text {lift }}(z) u^{a},
$$


where $X_{S} \in \mathcal{X}(E)$ is the drift vector field, the $Y_{a}^{\text {lift }} \epsilon$ $\mathcal{X}(E)$ are control vector fields, and the $u^{a}$ are inputs, for $a=1 \ldots m$. The vector fields must obey the 1 -homogeneity requirements. We consider the case $X_{S} \in \mathcal{P}_{1} \oplus \mathcal{P}_{-1} \subset \mathcal{M}_{1}$, which covers simple mechanical systems plus additional mechanical systems ${ }^{1}$. Consequently, $X_{S}$ can be written ${ }^{2}$ as,

$$
X_{S}=\Gamma-Z^{\mathrm{lift}},
$$

where $\Gamma \in \mathcal{P}_{1}$ and $Z^{\text {lift }} \in \mathcal{P}_{-1}$. The control vector fields must satisfy $Y_{a}^{\text {lift }} \in \mathcal{P}_{-1}$, as implied by the fact that they are lifts.

In what follows we will relate the control system (11) to free Lie algebras. Sussmann's work has demonstrated that much progress can be achieved by examining an algebraic abstraction of the control system. By placing the two descriptions in bijective correspondance, properties of the free Lie algebra translate to properties of the original control system. Geometric homogeneity fits within this algebraic realization; we need only examine how bracketing affects the homogeneous order of elements of free Lie algebras.

Free Lie algebras. Consider a finite set $\mathbf{X}=$ $\left\{X_{0}, X_{1}, \ldots, X_{l}\right\}$ of elements. The free Lie algebra, denoted $L(\mathbf{X})$, is the involutive closure of $\mathbf{X}$ under Jacobi-Lie brackets. Denote by $\operatorname{Br}(\mathbf{X})$ the subset of $L(\mathbf{X})$ containing only products (i.e. brackets) of elements in $\mathbf{X}$; this subset will generate $L(\mathbf{X})$ as a $\mathbb{R}$-vector space (see Prop. 10). Consequently, we need only consider the generating set, $B r(\mathbf{X})$ instead of the full set $L(\mathbf{X})$.

For each element $B \in B r(\mathbf{X})$, let $\delta_{a}(X)$ be the number of times that the element $X_{a}, a=0, \ldots, l$, occurs in $B$. The degree of $B$, denoted $\delta(B)$, is the sum of the $\delta_{a}$ 's: $\delta(B) \equiv$ $\delta_{0}(B)+\sum_{a=1}^{l} \delta_{a}(B)$. The relative degree, denoted by $\tilde{\delta}(\cdot)$, is the difference between $\delta_{0}$ and the sum of the $\delta_{a}$ 's for $a=$ $1 \ldots l: \tilde{\delta}(B) \equiv \delta_{0}(B)-\sum_{a=1}^{l} \delta_{a}(B)$.

Proposition 10 [3] Every element of $L(\mathbf{X})$ is a linear combination of repeated brackets of the form,

$$
\left[X_{k},\left[X_{k-1},\left[\ldots,\left[X_{2}, X_{1}\right] \ldots\right]\right\}\right]
$$

where $X_{i} \in \mathbf{X}, i=1, \ldots, k$.

Connection to Vector Fields. A family of vector fields on the manifold $Q$ is a subset $\mathcal{X} \subset \mathcal{X}(Q)$. The family may be used to define a distribution on $Q$,

$$
D_{\mathcal{V}}(x) \equiv \operatorname{span}_{\mathbb{R}}\{X(x) \mid X \in \mathcal{V}\}
$$

The smallest Lie subalgebra of the Lie algebra, $\mathcal{X}(Q)$, is the set of vector fields on $Q$ generated by repeated Lie brackets of elements in $\mathcal{V}$. This Lie subalgebra, which will be used for small-time controllability results, is generated by the free Lie algebras previously described.

Let $\mathrm{X}$ and $\mathcal{V}$ be in bijective correspondence via the bijection, $\phi: \mathbf{X} \rightarrow \mathcal{V}$. A Lie algebra homomorphism between $L(\mathbf{X})$ and $\mathcal{X}(Q)$, is defined by $\operatorname{Ev}(\phi): L(\mathbf{X}) \rightarrow \mathcal{X}(Q)$,

$$
\operatorname{Ev}(\phi), \quad u_{1} \otimes \cdots \otimes u_{k} \mapsto \phi\left(u_{1}\right) \cdot \cdots \cdot \phi\left(u_{k}\right),
$$

where the products, $\cdot$, correspond to Jacobi-Lie brackets. The smallest Lie subalgebra of $\mathcal{X}(Q)$ containing $\mathcal{V}$, is the image

\footnotetext{
${ }^{1}$ The full case $X_{S} \in \mathcal{M}_{1}$ can be found in [11].

${ }^{2}$ The minus sign in $X_{S}=\Gamma-Z^{\text {lift }}$ is an artifact of the simple mechanical systems paradigm, and comes from the Euler-Lagrange equations.
}

of $\operatorname{Ev}(\phi)(L(\mathbf{X}))$, which shall be denoted $\overline{\mathrm{Lie}}(\mathcal{V})$ and called the involutive closure of $\mathcal{V}$.

When evaluated at a point, $q \in Q$, the homomorphism $\operatorname{Ev}_{q}(\phi)$ maps $L(\mathbf{X})$ into $T_{q} Q$. The Lie algebra rank condition (LARC) at $q$ is satisfied if,

$$
\operatorname{Ev}_{q}(\phi)(L(\mathbf{X}))=T_{q} Q
$$

or, equivalently,

$$
\operatorname{rank}(\overline{\operatorname{Lie}}(\mathcal{V}))=\operatorname{dim} Q
$$

for $\phi: \mathbf{X} \rightarrow \mathcal{V}$, where $\mathcal{V}=\left\{f, g_{1}, \ldots, g_{m}\right\}$. LARC is used to determine local accessibility, from which small-time local controllability may follow under additional requirements. The condition still applies if $Q$ is the vector bundle $E$.

Properties of and Computations with the Free Lie Algebra. The sets that are typically placed into bijective correspondence are $\mathbf{X}^{\prime}$ and $\mathcal{V}^{\prime}=\left\{X_{S}, Y_{1}^{\text {lift }}, \ldots, Y_{m}^{\text {tift }}\right\}$, Eq. (11). The bijection will take $X_{0}^{\prime}$ to $X_{S} \in \mathcal{M}_{1}$, and $X_{a}^{\prime}$ to $Y_{a}^{\text {lift }} \in \mathcal{M}_{-1}, a=1 \ldots m$. Instead, decompose the drift $X_{S}$ into its two homogeneous components, Eq. (12). The sets in bijective correspondence will be $\mathbf{X}$ and $\mathcal{V}=$ $\left\{\Gamma, Y_{1}^{\text {lift }}, \ldots, Y_{m}^{\text {lift }}, Z^{\text {lift }}\right\}$. Now, $X_{0}$ will map to $\Gamma \in \mathcal{P}_{1}$, and the $X_{a}$ will map to $Y_{a}^{\text {lift }}$ for $a=1 \ldots m$, and $X_{m+1}$ will map to $Z^{\text {lift }}$, all elements off $\mathcal{P}_{-1}$. The link between the two sets, $\mathbf{X}$ and $\mathbf{X}^{\prime}$, will be discussed shortly.

Define the following notation,

$$
\begin{aligned}
& B r^{k}(\mathbf{X})=\{B \in B r(\mathbf{X}) \mid \delta(B)=k\} \\
& B r_{k}(\mathbf{X})=\{B \in B r(\mathbf{X}) \mid \tilde{\delta}(B)=k\} .
\end{aligned}
$$

$B r^{k}(\mathbf{X})$ is the set of elements in $B r(\mathbf{X})$ whose degree is - $k$, whereas $B r_{k}(\mathbf{X})$ is the set of elements in $B r(\mathbf{X})$ whose relative degree is $k$.

Definition 16 [3] Let $B \in B r_{0}(\mathbf{X}) \cup B r_{-1}(\mathbf{X})$ and let $B_{1}, B_{2}, B_{11}, B_{12}, B_{21}, B_{22}, \ldots$ be the component decomposition of $B$. We shall say that $B$ is primitive if each of its components is in $B r_{-1}(\mathbf{X}) \bigcup B r_{0}(\mathbf{X}) \bigcup\left\{X_{0}\right\}$.

Some observations may be made about primitive brackets.

Observation 1 [3] If $B \in B r_{-1}$ is primitive, then, up to sign, we may write $B=\left[B_{1}, B_{2}\right]$ with $B_{1} \in B r_{-1}(\mathbf{X})$ and $B_{2} \in B r_{0}(\mathbf{X})$.

Observation 2 [3] If $B \in B r_{0}(\mathbf{X})$ is primitive, then, up to sign, $B$ may have one of two forms. Either $B=\left[X_{0}, B_{1}\right]$ with $B_{1} \in B r_{-1}(\mathbf{X})$ printitive, or $B=\left[B_{1}, B_{2}\right]$ with $B_{1}, B_{2} \in$ $B r_{0}(\mathbf{X})$ primitive.

Lemma 1 [3] Let us impose the condition on elements of $B r(\mathbf{X})$ that we shall consider a bracket to be zero if any of its components are in $B r_{-j}(\mathbf{X})$ for $j \geq 2$. Let $B \in$ $B r_{0}(\mathbf{X}) \bigcup B r_{-1}(\mathbf{X})$. Then $B$ is the finite sum of primitive brackets.

Observation 3 [II] If $B \in B r_{0}(\mathbf{X})$, then $B$ may be written as $\left[X_{0}, B_{1}\right]$, with $B_{1} \in B r_{-1}(\mathbf{X})$, or as the sum of repeated brackets of such forms, i.e., elements that look like

$$
\left[C_{i},\left\{C_{i-1},\left[\ldots,\left[C_{2}, C_{1}\right] \ldots\right]\right]\right]
$$

where the $C_{i} \in B r_{0}(\mathrm{X})$ are, up to sign, of the form $\left[X_{0}, C_{i 1}\right]$ with $C_{i 1} \in B r_{-1}(\mathbf{X})$. 
The set $\mathbf{X}$, under the evaluation map $\operatorname{Ev}(\cdot)$, does not correspond to the vector fields found in equation (11); the proper set to use is $\mathbf{X}^{\prime}$. A method to equate an element of $B r\left(\mathbf{X}^{\prime}\right)$ to elements of $B r(\mathbf{X})$ must be given. This will require defining the mapping $\Phi$ and the set $\mathbf{S}(\cdot) \subset B r(\mathbf{X})$. Suppose that $B^{\prime} \in B r\left(\mathbf{X}^{\prime}\right)$, then given that $\mathbf{X}$ maps onto $\mathbf{X}^{\prime}$, it is possible to write $B^{\prime}$ as a $\mathbb{R}$-linear sum of elements in $\operatorname{Br}(\mathbf{X})$. These primitive elements define the set $\mathbf{S}\left(B^{\prime}\right)$.

Lemma 2 [3] The mapping $\Phi: B r\left(\mathbf{X}^{\prime}\right) \rightarrow L(\mathbf{X})$ is,

$$
\Phi\left(B^{\prime}\right)=\sum_{B \in \mathbf{S}\left(B^{\prime}\right)}(-1)^{\delta_{n+1}(B)} B, \text { for } B^{\prime} \in B r\left(\mathbf{X}^{\prime}\right) .
$$

Intuitively, the element $B^{\prime} \in B r\left(\mathbf{X}^{\prime}\right)$ is converted to a sum of elements in $\operatorname{Br}(\mathbf{X})$ by replacing $X_{0}^{\prime}$ with $X_{0}-X_{m+1}$ and equating $X_{a}^{\prime}$ with $X_{a}$ for $a=1 \ldots m$, then using the multi-linearity of the iterated Jacobi-Lie bracket to obtain a sum of Jacobi-Lie brackets, which comprise the set $\mathbf{S}\left(B^{\prime}\right) \subset$ $\operatorname{Br}(\mathbf{X})$. A simple example is the following,

$$
\begin{aligned}
{\left[X_{1}^{\prime},\left[X_{0}^{\prime}, X_{2}^{\prime}\right]\right] } & =\left[X_{1},\left[X_{0}-X_{m+1}, X_{2}\right]\right] \\
& =\left[X_{1},\left[X_{0}, X_{2}\right]\right]-\left[X_{1}:\left[X_{m+1}, X_{2}\right]\right]
\end{aligned}
$$

The two iterated Jacobi-Lie brackets, $\left[X_{1},\left[X_{0}, X_{2}\right]\right]$ and $\left[X_{1},\left[X_{m+1}, X_{2}\right]\right]$, form the set $\mathbf{S}\left(B^{\prime}\right)$, where the sign of their contribution is related to the degree $\delta_{m+1}(\cdot)$.

Free Symmetric algebras. Consider the finite set $\mathbf{Y}=$ $\left\{X_{1}, \ldots, X_{l+1}\right\}$. The free symmetric algebra, denoted $S(\mathbf{Y})$, generated by $\mathbf{Y}$ is the closure under the symmetric product,

$$
\left\langle X_{a}: X_{b}\right\rangle \equiv\left[X_{b},\left[X_{0}, X_{a}\right]\right] \text { : }
$$

Denote by $\operatorname{Pr}(\mathbf{Y})$ the subset of $S(\mathbf{Y})$ containing only products (i.e. symmetric products) of elements in $\mathbf{Y}$. Let $\gamma_{a}(X)$ be the number of times that the element $X_{a}, a=1, \ldots, l+1$, occurs in $P \in \operatorname{Pr}(\mathbf{Y})$. The degree of $P \in \operatorname{Pr}(\mathbf{Y})$ denoted $\gamma(\cdot)$, is the sum of the $\gamma$ 's, $\gamma(P)=\sum_{a=1}^{l+1} \gamma_{a}(P)$, where $X_{0} \in \mathbf{X}$ maps to $\Gamma \in \mathcal{X}(E)$ under $\phi$.

The set $\mathrm{Y}$ will be bijective to $\mathcal{Y}=\left\{Y_{1}^{\text {lift }}, \ldots, Y_{l}^{\text {lift }}, Z^{\text {lift }}\right\}$ via the bijection $\psi: \mathbf{Y} \rightarrow \mathcal{Y}$. The bijection $\psi$ may be extended naturally to obtain a homomorphism from $S(\mathbf{Y})$ to $\mathcal{X}(E)$. When evaluated for $P \in S(\mathbf{Y})$, at a point $x \in E$, the map that results, denoted by $\operatorname{Ev}(\psi)$, is

$$
\operatorname{Ev}_{x}(\psi)(P)=(\operatorname{Ev}(\psi)(P))(x)
$$

Consequently, the symmetric closure may be defined to be the image under the free symmetric algebra,

$$
\overline{\operatorname{Sym}}(\mathcal{Y}) \equiv \operatorname{Im}(\operatorname{Ev}(\psi)(S(\mathbf{Y}))) .
$$

Properties of and Computations with the Free Symmetric Algebra The set used to create the free symmetric algebra, $Y$, is really a subset of $X$, the set used to create the free Lie algebra. By definition of the product used in the free symmetric algebra, its properties translate to the appropriate subset of the free Lie algebra.

Observation 4 [11] Suppose that $B$ is a primitive. If $B \in$ $B r_{-1}(\mathbf{X})$, then $B \in S(\mathbf{Y})$.

This observation is analogous to Lemma 5.6 part (i) of [3].

3.1.1 Free Algebras and Homogeneity: Here we relate the algebraic property of relative degree to geometric homogeneity, and examine the consequences.
Lemma $3[11]$ Let $l \geq 1$ be an integer and let $B \in B r_{l}(\mathbf{X})$. Then $\operatorname{Ev}(\phi)(B)\left(0_{q}\right)=0$ for all $q \in Q$.

Proof: The relative degree gives the homogeneous order of the resulting vector field. When evaluated on the zero section, homogeneity of order $l \geq 1$ implies that the result is the zero vector, c.f. Proposition 3.

Lemma 4 Let $l \geq 2$ be an integer, and let $B \in B r_{-l}(\mathbf{X})$. Then $\operatorname{Ev}(\phi)(B)=0$.

Proof: For $l \geq 2$, the space only contains the zero vector field, c.f. Proposition 2.

The homogeneous structure of the nonlinear system implies that only the brackets that reside in $\mathcal{P}_{0} \oplus \mathcal{P}_{-1}$ when evaluated on the zero section of $E$, will give contributions to the controllable Lie subalgebra. The homogeneous structure also implies that the brackets of $\mathcal{P}_{-1}$ will be vertical elements, and the brackets of $\mathcal{P}_{0}$ evaluated on the zero section will be horizontal elements, using the trivial connection.

3.1.2 STLC in the algebraic sense: In order to determine STLC using the Lie algebra rank condition, Sussmann needed the notion of a good and a bad bracket.

Definition 17 [2] An element $B \in B r(\mathbf{X})$ is called bad if $\delta_{a}(B)$ is even for all $a=1 \ldots m$, and if $\delta_{0}(B)$ is odd. If $B$ is not bad, then it is good.

In addition, Sussmann utilized the permutation group $S_{m}$, of permutations using $m$ symbols. An element $\sigma \in S_{m}$ takes the $m$ elements $X_{a} \in \mathbf{X}$, and maps them to $X_{\sigma(a)} \in \mathbf{X}$. The set of all possible permutations can be used to define a bracket permutation, $\beta: B r(\mathbf{X}) \rightarrow B r(\mathbf{X})$ for $B \in B r(\mathbf{X})$.

$$
\beta(B)=\sum_{\sigma \in S_{m}} \sigma(B)
$$

Sussman's general derivation, relying on the standard form of the nonlinear control equation (8), culminated in the theorem,

Theorem 1 [2] Let $\phi: \mathbf{X} \rightarrow\left\{f, g_{1}, \ldots, g_{m}\right\}$, be a bijection sending $X_{0}$ to $f$ and $X_{a}$ to $g_{a}$ for $a=1 \ldots m$. Suppose that $(8)$ is such that every bad bracket $B \in B r(\mathrm{X})$ has the property that

$$
\operatorname{Ev}_{z}(\phi)(\beta(B))=\sum_{a=1}^{k} \xi_{a} \operatorname{Ev}_{z}(\phi)\left(C_{a}\right)
$$

where $C_{a}$ are good brackets in $B r(\mathbf{X})$ of lower degree than $B$ and $\xi^{a} \in \mathbb{R}$ for $a=1 \ldots k$. Also suppose that (8) satisfies the LARC at $z$. Then (8) is STLC at $z$.

Lewis examined the structure of simple mechanical systems to refine this idea for configuration controllability. In particular, only the brackets in the homogeneous space $B r_{-1}(\mathbf{X})$ can result in bad brackets. The search for bad brackets is reduced to examination of the symmetric products only. Define $\psi$ to be the bijection $\mathbf{Y}=\left\{X_{1}, \ldots, X_{m+1}\right\} \mapsto \mathcal{Y}$.

Definition 18 An element $P \in \operatorname{Pr}(\mathbf{Y})$ is called bad if $\gamma_{a}(P)$ is even for each $a=1 \ldots m$. If $P$ is not bad then it is good.

Theorem 2 [3] Consider the bijection $\psi: \mathbf{Y} \rightarrow \mathcal{Y}$, which sends $X_{a}$ to $Y_{a}^{\text {lift }}$ for $a=1 \ldots m$, and $X_{l+1}$ to $Z^{\text {lift }}$. Suppose that (II) is such that every bad symmetric product in $P \in$ $\operatorname{Pr}(\mathbf{Y})$ has the property that

$$
\operatorname{Ev}_{0_{x}}(\psi)(\rho(P))=\sum_{a=1}^{k} \xi_{a} \operatorname{Ev}_{0_{x}}(\psi)\left(C_{a}\right)
$$


where $C_{a}$ are good synmetric products in $\operatorname{Pr}(\mathbf{Y})$ of lower degree than $P$ and $\xi_{a} \in \mathbb{R}$ for $a=1, \ldots, k$. Also, suppose that (11) is locally configuration accessible at $x$. Then (11) is STLCC at $x$.

This theorem was proven for the case that $E=T Q$, and is precisely the theorem that will be recreated for a 1homogeneous control system evolving on a generic vector bundle Configuration controllability will imply controllability of the base space, $M$, of the vector bundle, $E$.

\subsection{Conditions for Configuration Controllability}

Up to this point, we have given and proven the analogous theorems derived in [3] for use within the context of 1homogeneous control sytems. Consequently, all of the theorems concerning configuration controllability may now hold for these homogeneous control sytems without modification of the proofs from Lewis and Murray [3]. As configuration controllability analysis occurs on the zero section of $T E$, we need some preliminary results characterizing the zero section.

Lemma 5 Let $x \in M$. Then,

$$
\begin{aligned}
& D_{\overline{\operatorname{Lie}(\mathcal{V})}}\left(0_{x}\right) \bigcap V_{0_{x}} E=D_{\overline{S_{y m}(\mathcal{Y})}}\left(0_{x}\right) \text {, and } \\
& D_{\overline{\operatorname{Lie}}(\mathcal{V})}\left(0_{x}\right) \cap H_{0_{x}} E=D_{\overline{\operatorname{Lie}}([\Gamma, \overline{\operatorname{Sim}}(\mathcal{Y})])}\left(0_{x}\right)
\end{aligned}
$$

Lemma 5 allows us to compare the involutive closures on the right hand side with the horizontal and vertical distributions of the trivial connection to come up with equivalent statements for the free Lie algebra, $L(\mathbf{X})$. In the presence of a nonvanishing potential, additional work is needed to demonstrate that the Lie algebra $L\left(\mathbf{X}^{\prime}\right)$ is represented by $L(\mathbf{X})$, or equivalently $\operatorname{Br}\left(\mathbf{X}^{\prime}\right)$ is represented by $B r(\mathbf{X})$. The brackets in $B r(\mathbf{X})$ neeed to be equated with brackets in $B r\left(\mathbf{X}^{\prime}\right)$, which is done via the replacement operator,

Definition 19 The replacement operator, $\operatorname{Rep}: B r(\mathbf{X}) \rightarrow$ $B r\left(\mathbf{X}^{\prime}\right)$, operates as follows: given an element $B \in B r(\mathbf{X})$, all instances of $X_{0} \in \mathbf{X}$ and $X_{m+1} \in \mathbf{X}$ are replaced with $X_{0}^{\prime} \in \mathbf{X}^{\prime}$, and all instances of $X_{a}$ are replaced with $X_{a}^{\prime}$ for $a=1 \ldots m$.

The term $X_{m+1}$ evaluates to the vector field $Z^{\text {lift }}$ which cannot occur on its own, it is part of the drift vector field. The replacement map respects this fundamental fact. Define the function $\tilde{\Phi}$ to map $B r(\mathbf{X})$ into $\operatorname{Br}\left(\mathbf{X}^{\prime}\right)$, then finally into $L(\mathbf{X})$.

$$
\widetilde{\Phi}: B r(\mathbf{X}) \rightarrow L(\mathbf{X}), \quad \widetilde{\Phi} \equiv \Phi \circ \operatorname{Rep}
$$

Definition 20 The horizontal and vertical distributions corresponding to $\mathrm{Br}^{k}\left(\mathbf{X}^{\prime}\right)$ are,

$$
\begin{aligned}
& \mathcal{C}_{\text {hor }}^{(k)}\left(\mathcal{V}^{\prime}\right)=\{\operatorname{Ev}(\phi)(\widetilde{B}) \mid \widetilde{B}=\tilde{\Phi}(B), \\
&\left.\forall B \in B r^{k}(\mathbf{X}) \bigcap B r_{0}(\mathbf{X})\right\} \\
& \mathcal{C}_{\text {ver }}^{(k)}\left(\mathcal{V}^{\prime}\right)=\{\operatorname{Ev}(\phi)(\tilde{B}) \mid \widetilde{B}=\tilde{\Phi}(B), \\
&\left.\forall B \in B r^{k}(\mathbf{X}) \bigcap B r_{-1}(\mathbf{X})\right\}
\end{aligned}
$$

We may now define the horizontal and vertical distributions by taking the union over all $k \in \mathbb{Z}^{+}$.
Definition 21 The horizontal and vertical distributions are defined to be,

$$
\begin{gathered}
\mathcal{C}_{\text {hor }}\left(\mathcal{V}^{\prime}\right)=\bigcup_{k \in \mathbf{Z}^{+}} \mathcal{C}_{\text {hor }}^{(k)}\left(\mathcal{V}^{\prime}\right), \text { and } \\
\mathcal{C}_{\text {ver }}\left(\mathcal{V}^{\prime}\right)=\bigcup_{k \in \mathbb{Z}^{+}} \mathcal{C}_{\text {ver }}^{(k)}\left(\mathcal{V}^{\prime}\right) .
\end{gathered}
$$

All of the fundamental work to prove the following statement has been completed.

Proposition 11 Let $x \in M$. Then,

$$
\begin{gathered}
D_{\overline{\operatorname{Lie}}\left(\mathcal{V}^{\prime}\right)}\left(0_{x}\right) \bigcap H_{0_{x}} E=C_{\text {hor }}\left(\mathcal{V}^{\prime}\right)\left(0_{x}\right), \quad \text { and } \\
D_{\overline{\operatorname{Lie}}\left(\mathcal{V}^{\prime}\right)}\left(0_{x}\right) \bigcap V_{0_{x}} E=C_{v e r}\left(\mathcal{V}^{\prime}\right)\left(0_{x}\right) .
\end{gathered}
$$

Configuration controllability tests may now follow.

Theorem 3 [3] The control system (11) is locally configura. tion accessible at $x \in M$ if $\mathcal{C}_{\text {hor }}\left(\mathcal{V}^{\prime}\right)\left(0_{x}\right)=H_{0_{x}} E$.

The partial converse proven for the case when there is no potential also holds.

Theorem 4 Suppose that $Z^{\text {lift }}=0$ and (11) is locally configuration accessible. Then $\mathcal{C}_{\text {hor }}\left(\mathcal{V}^{\prime}\right)\left(0_{q}\right)=H_{0_{q}} E$ for $Q$ in an open dense subset of $Q$.

Theorem 5 Suppose that $\mathcal{Y}$ is such that every bad symmetric product in $\operatorname{Pr}(\mathbf{Y})$ has the property that

$$
\operatorname{Ev}_{0_{x}}(\psi)(\rho(P))=\sum_{a=1}^{m} \xi_{a} E_{0_{0}}(\psi)\left(C_{a}\right)
$$

where $C_{a}$ are good symmetric products in $\operatorname{Pr}(\mathbf{Y})$ of lower degree that $P$ and $\xi_{a} \in \mathbb{R}$ for $a=1 \ldots m$. Also, suppose that (II) is locally configuration accessible at $x \in M$. Then (II) is STLCC at $x$.

Corollary 4 Suppose that the hypotheses of Thm. 5 hold for all $x \in M$. Then the system (JI) is equilibrium controllable.

\section{Examples}

In this section, we will take various known analyses of controllability for choices of the vector bundle $E$.

The Tangent Bundle. Let $E=T M$. In a local trivialization, the second iterated tangent bundle takes the form $T(T Q) \cong Q \times E \times E \times E$. Thus both the vertical and horizontal subspaces of $T(T Q)$ can be identified with $T Q$. This fact lies behind much of the simplification that occurs in [3]. In particular,

$$
\left[\Gamma, X_{a}^{\mathrm{lift}}\right] \in H E \quad \text { and } \quad T \pi\left(\left[\Gamma, X_{a}^{\mathrm{lift}}\right]\right)=-X_{a} .
$$

Consequently, $\left[\left[\Gamma, X_{a}^{\text {lift }}\right],\left[\Gamma, X_{b}^{\text {lift }}\right]\right] \leftrightarrow\left[X_{a}, X_{b}\right]$, where the Jacobi-Lie bracket of the right hand side is of vector fields in $\mathcal{X}(M)$ instead of $\mathcal{X}(T M)$, meaning that the space $\overline{\mathrm{Lie}}([\Gamma, \overline{\operatorname{Sym}}(\mathcal{Y})])$ can now be given by $\overline{\mathrm{Lie}}(\tilde{\mathcal{Y}})$, where the set $\tilde{\mathcal{Y}}$ is uniquely defined by $\widetilde{\mathcal{Y}}^{\text {lift }} \equiv \mathcal{Y}$. Additionally there exists a symmetric product defined on $\mathcal{X}(M)$ such that the operation of vertical lifting commutes with the symmetric product. The space $\overline{\operatorname{Sym}}(\mathcal{Y})$ can now be given by $\overline{\operatorname{Sym}}(\widetilde{\mathcal{Y}})$.

Essentially, all of the calculations drop to $T M$, resulting in computational and notational reductions, precisely as emphasized by Lewis and Murray [3]. Many known canonical forms are instances of this type. Given a second order vector field, $X \in \mathcal{X}(T Q)$, if it is of homogeneous order +1 , it is said to be 
a spray [10]. There exists a bijection between linear symmetric connections and sprays (an affine connection and its related Christoffel symbols form such a bijection); see $[9,10]$. Therefore, controllability analysis of any second order vector field such that $X_{S} \in\left(\mathcal{P}_{1} \oplus \mathcal{P}_{-1}\right)$ is of this type.

The Cotangent Bundle. Controllability analysis can be performed on the cotangent bundle just as easily as on the tangent bundle. A notable exception is that the identification of the horizontal and vertical tangent spaces of $T\left(T^{*} Q\right)$ with the covector bundle $T^{*} Q$ is not made. Analysis on the cotangent bundle is useful for Hamiltonian systems, although we are unaware of any research that studies how the Lie-algebraic structure of Hamiltonian systems may be used for control.

Constrained Mechanical Systems. Controllability for systems with nonholonomic velocity constraints can be addressed using this theory. While affine connections and constraints are compatible, we focus on another approach that describe constraints via an Ehresmann connection, $A: T Q \rightarrow$ $V Q$, on a fiber bundle $Q$ with bundle projection $\tau: Q \rightarrow M$ and model fiber $S$ [13]. The appropriate vector bundle is $E \cong M \times S \times V$, where $V$ is the model vector space for the tangent bundle $T M$, and $M \times S$ is the base space. Configuration controllability uses $\overline{\operatorname{Lie}}([\Gamma, \overline{\operatorname{Sym}}(\mathcal{Y}])$, see [5].

In this example the tangent bundle $T E$ cannot be identified with $E$, so simplification does not occur. However, the configuration controllability test is equivalent to taking Lie brackets of horizontally lifted vector fields in $\mathcal{X}(M)^{3}$, which is equivalent to having the local curvature form, $B_{l o c}(r, s)$, of the Ehresmann connection, and its higher-order covariant derivatives. Constraint reduction simplifies the controllability analysis to the connection form only.

If the fiber is a Lie group, $S=G$, and the equations of motion are group invariant ${ }^{4}$, then we are in the principal bundle case. The local form of the principal connection, $\mathcal{A}_{\text {loc }}(r): T_{r} M \rightarrow \mathfrak{g}$, only depends on the base space. Configuration controllability is further reduced to analysis of the associated adjoint bundle $\tilde{\mathfrak{g}}$, recovering $[14,15]$.

Constrained Mechanical Systems with Symmetry For some systems, the constraints do not span the Lie algebra, $\mathfrak{g}$, of the Lie group, $G$, meaning that not all of the fiber is constrained. Let the control system in question evolve on a principal bundle $\pi: Q \rightarrow M$, with Lie group $G$. The system will evolve on the vector bundle $E \cong T M \times G \times V$, with base diffeomorphic to $M \times G$, and vector bundle diffeomorphic to $W \times V$, where $W$ is the model fiber for the tangent space $T M$. Typically, the vector space $V$ will be either the Lie algebra $\mathfrak{g}$, or its dual $\mathfrak{g}^{*}$. The homogeneous analysis will recover the controllability results from both instances. For $V=\mathfrak{g}$, this recovers the work of Cortés et al. [4], and for $V=\mathfrak{g}^{*}$ this recovers Ostrowski and Burdick [6].

\section{Conclusion}

This paper explored the role of geometric homogeneity on the evolution of control systems on vector bundles and developed a controllability a test analagous to those for simple mechanical systems, but at a more general level. Additional accessibility tests are given in [11], as configuration control does not suffice for all types of mechanical systems.

It would clearly be useful to design control laws from the controllability analysis. This has been done in the simple mechanical system framework by Bullo [16]. In related work, the authors have developed a generalized averaging method for nonlinear systems that recovers the synmetric products and Lie brackets needed for control. This technique generalizes the body of work on motion control algorithms to arbitrary 1-homogeneous control systems, just as this paper generalizes the body of work on controllability for simple mechanical systems. A companion paper discusses this technique [17].

\section{References}

11] F. Bullo and A. D. Lewis. On the homogeneity of the affine connection model for mechanical systems. In Proc. IEEE Conf. Dec. Contr, pages 1260-1265, 2000.

[2] H. J. Sussmann. A general theorem on local controllability. SIAM J. Contr. Opt., 25(1), 1987.

[3] A. D. Lewis and R. M. Murray. Configuration controllability of simple mechanical control systems. SIAM J. Contr. Opt., 35(3):766-790, 1997.

[4] J. Cortés, S. Martínez, J. P. Ostrowski, and H. Zhang. Simple mechanical control systems with constraints and symmetry. SIAMJ. Contr. Opt., 41(3):851-874, 2002.

15] A. M. Bloch, M. Reyhanoglu, and N. H. McClamroch. Control and stabilization of nonholonomic dynamic systems. IEEE Trans. Aut. Contr., 37(11):1746-1757, 1992.

[6] J. Ostroswki and J. Burdick. Controllability tests for mechanical systems with constraints and symmetries. J. Appl. Math. and Comp. Sci., 7(2):101-127, 1997.

[7] M. Kawski. Geometric homogeneity and applications to stabilization. In A. Krener and D. Mayne, editors, Proc. IFAC Conf. Nonlin. Contr. Sys. Des., pages 147-152, Tahoe City, CA, 1995.

[8] R. M'Closkey and P. Morin. Time-varying homogeneous feedback: Design tools for the exponential stabilization of systems with drift. Int. J. Cont., 71(5):837-869, 1998.

[9] M. Crampin. Tangent bundle geometry for Lagrangian dynamics. J. Phys. A, 16:3755-72, 1983.

[10] I. Kolár, P. Michor, and J. Slovák. Natural Operations in Differential Geometry. Springer-Verlag, Berlin, 1993.

[11] P. A. Vela and J. W. Burdick. Geometric homogeneity and controllability of nonlinear systems. available electronically http:www:cds.caltech.edw/pvela, 2003.

[12] P. E. Crouch. Geometric structures in systems theory. IEE Proc. Part D, Control Theory and Applications, 128(5), 1981.

[13] A. M. Bloch, P. S. Krishnaprasad, J. E. Marsden, and R. M. Murray. Nonholonomic mechanical systems with symmetry. Arch. Rat. Mech. Anal., 136:21-99, 1996.

[14] S. D. Kelly and R. M. Murray. Geometric phases and robotic locomotion. Journal of Robotic Systems, 12(6):417-431, 1995.

[15] J. E. Radford and J. W. Burdick. Local motion planning for nonholonomic control systems evolving on principal bundles. Proc. IEEE Int. Conf. Rob. Aut., 1999.

[16] F. Bullo. Nonlinear Control of Mechanical Systems: A Riemannian Geometry Approach. PhD thesis, Caltech, 1999.

[17] P. A. Vela and J. W. Burdick. Control of mechanical systems with drift using higher-order averaging. Submitted IEEE Conf. Dec. Contr., 2003.

\footnotetext{
"The horizontal lift is using the constraint connection form.

${ }^{4}$ Typically this arises from group invariant Lagrangian and constraints.
} 